\title{
Velutin, an Aglycone Extracted from Korean Mistletoe, with Improved Inhibitory Activity against Melanin Biosynthesis
}

\author{
Se-Hui Jung ${ }^{1,+} \mathbb{\oplus}$, Jaehyun Kim ${ }^{1,+}$, Juneyong Eum ${ }^{2}$, Jung Won Choe ${ }^{1}$, Hak Hyun Kim ${ }^{1}$, \\ Yun Kee ${ }^{2, *(\mathbb{D})}$ and Kooyeon Lee ${ }^{1, *}$ \\ 1 Department of Bio-Health Technology, College of Biomedical Science, Kangwon National University, \\ Chuncheon 24341, Korea \\ 2 Division of Biomedical Convergence, College of Biomedical Science, Kangwon National University, \\ Chuncheon 24341, Korea \\ * Correspondence: yunkee@kangwon.ac.kr (Y.K.); lky@kangwon.ac.kr (K.L.); Tel.: +82-33-250-8384 (Y.K.); \\ +82-33-250-6477 (K.L.) \\ + These authors contributed equally to this work.
}

Academic Editors: Celestino Santos-Buelga and Ana M. González-Paramás Received: 16 June 2019; Accepted: 11 July 2019; Published: 12 July 2019

\begin{abstract}
The abnormal regulation of melanin synthesis leads to a wide range of pigmentary disorders. Although various melanin biosynthesis inhibitors have been developed, their efficacy and long-term safety needs to be further improved, and thus the goal of this study is to develop promising natural compound inhibitors of melanin biosynthesis. Here, we obtained aglycone flavonoid extract through the microwave-assisted hydrolysis of glycoside extract from Korean mistletoe in acidic condition. The aglycone extract inhibited tyrosinase activity more efficiently with better antioxidant activity than glycoside extract in vitro. The microwave-assisted aglycone extract of mistletoe was further analyzed for in vivo activity, and the results showed the aglycone extract inhibited both early melanocyte development and melanin synthesis more efficiently in zebrafish embryo in a dose-dependent manner. Our in vivo toxicity assay quantitatively measured cell death in zebrafish embryos and showed that the microwave-assisted aglycone extract of mistletoe had no significant effect on cell death $(p<$ 0.001 ), indicating that aglycone extract is more biocompatible than glycoside extract. Furthermore, our in vitro and in vivo analyses successfully identified and characterized velutin, an aglycone of a homoflavoyadorinin B glycoside, as a major inhibitory component in the microwave-assisted mistletoe extract. Ultimately, this study showed that the novel natural compound inhibitor velutin, which was generated through microwave-assisted extraction from mistletoe, improved the efficacy of melanin biosynthesis inhibition with little toxicity.
\end{abstract}

Keywords: microwave-assisted hydrolysis; flavonoid aglycone extract; mistletoe; tyrosinase activity; velutin; zebrafish

\section{Introduction}

Melanin is a natural pigment that is synthesized in melanocytes through a multistep process, and it is involved in the determination of skin and hair color [1,2]. Melanin defends cells against harmful light such as UV; however, the abnormal regulation of melanin production is closely related to pigmentary disorders including vitiligo, pityriasis alba, and hyperpigmentation [3]. Melanin synthesis is regulated by multistep catalytic reactions of enzymes such as tyrosinase, tyrosine hydroxylase isoform I, phenylalanine hydroxylase, tyrosinase-related protein 1 , and tyrosinase-related protein 2 [4]. Tyrosinase is a copper-containing enzyme that plays a key role in the rate-limiting step of melanin synthesis by the hydroxylation of L-tyrosine to L-3,4-dihydroxyphenylalanine (L-DOPA) 
and subsequent oxidation of L-DOPA to $o$-quinone (dopaquinone) [5]. Therefore, tyrosinase is a target enzyme for the development of functional cosmetic agents and therapeutics against pigmentary disorders. Certain molecules obtained through chemical synthesis and natural resources, such as hydroquinone, azelaic acid, arbutin, and kojic acid, have been reported to prevent skin pigmentation by inhibiting tyrosinase activity $[4,6]$. However, due to the cytotoxicity, low efficacy, and low stability of the existing substances, there is still a need for natural substances with improved efficacy and safety.

Flavonoids are a large family of phenolic compound and widely present in the flowers, seeds, leaves, stems, and bark of plants. Flavonoids have emerged as attractive molecules in functional cosmetic ingredients due to their wide range of beneficial biological activities, including their antioxidant, anti-inflammatory, and antimicrobial activities $[7,8]$. In nature, most flavonoid compounds exist mostly as glycosides, as well as in the form of aglycones [9]. Although it requires further verification, it has been shown that the deglycosylation of flavonoid glycosides to their aglycones through the use of enzymes, heating, and microwave-irradiation improved their biological activities [10-13]. Microwave-assisted hydrolysis has emerged as a promising technology for the hydrolysis of glycoside extract due to its shorter reaction time and higher efficiency at low cost [14].

Mistletoe (Viscum album var. coloratum) is a hemiparasitic plant that derives nutritional requirements from oak trees, nettle trees, chestnut trees, as well as others as hosts [15,16]. Mistletoe contains a wide variety of chemical compounds including lectins, viscotoxins, polyphenols, and terpenoids, which have been widely applied as ingredients in herbal medicine throughout Europe and East Asia due to their beneficial biological activities, such as their anticancer, antidiabetic, antihypertensive, and antimelanogenesis activities [13,16,17]. In particular, viscumneoside III and homoflavoyadorinin B, are two flavonoid glycosides that have been extracted from Korean mistletoe and shown inhibitory effects on tyrosinase activity [16]. However, the anti-tyrosinase activity of flavonoid aglycones, which are deglycosylated from flavonoid glycosides of mistletoe, has yet to be examined.

Zebrafish have recently emerged as an attractive animal model for studying melanogenesis [18,19]. This is due to the fact that, in the embryonic development of zebrafish, tyrosinase gene expression and enzyme activity develop within $21 \mathrm{~h}$ post-fertilization and melanocytes start to appear from $24 \mathrm{~h}$ [20]. Directive 2010/63/EU, an animal protection law established by European Union, allowed for zebrafish larvae to be used for research or toxicity testing up to $96 \mathrm{~h}$ post-fertilization (hpf) in embryonic development, which increased the value of the zebrafish model in academic research and commercial development [21,22]. The European Union banned cosmetics-related research involving experimentation on animals through EC Regulation 1223/2009, making zebrafish even more attractive in the cosmetics industry as a resource to replace mammalian animal models $[18,19]$.

In this study, we prepared flavonoid aglycone extract through the microwave-assisted deglycosylation of flavonoid glycosides in crude Korean mistletoe extract under acidic condition and investigated the inhibitory effect of the aglycone extract on melanin biosynthesis in a zebrafish animal model. We identified velutin as a major natural compound inhibitor from the extract and further characterized its effect on melanin biosynthesis inhibition.

\section{Results and Discussion}

\subsection{Preparation of Flavonoid Aglycon Extract by Microwave-Assisted Hydrolysis of Flavonoid Glycosides}

In order to assess the potential improvement of natural compound inhibitors in Korean mistletoe, crude ethanol (glycosides-rich) extract of mistletoe was first prepared and further processed through microwave-assisted hydrolysis. The initial HPLC analysis of the crude ethanol extract revealed that viscumneoside III and homoflavoyadorinin B were the two major compounds at the retention times of 15.923 and $33.010 \mathrm{~min}$, respectively (Figure 1A). Next, we optimized the reaction condition of microwave-irradiation for reaction time, temperature, irradiation power, and $\mathrm{pH}$ regarding the deglycosylation of flavonoid glycosides in the extract. The crude EtOH extract was irradiated by 
microwave at 100,120 , or $150^{\circ} \mathrm{C}$ at $100 \mathrm{~W}$ for $1 \mathrm{~h}$ and then analyzed through HPLC under the conditions presented in Table 1 . Following irradiation at $120^{\circ} \mathrm{C}$, two major compounds were eluted at an increased retention time (Figure $1 \mathrm{~B}$ ), indicating that the polarities of the two compounds were changed by deglycosylation at $120^{\circ} \mathrm{C}$. The addition of acetic acid to the irradiation reaction increased the deglycolysis rate in a concentration-dependent manner (data not shown). Moreover, the two major compounds produced by microwave-irradiation were eluted at the same retention times as homoeriodictyol and velutin (Figure 1C), which are the deglycosylated forms of viscumneoside III and homoflavoyadorinin $B$, respectively. Here, we optimized the reaction conditions of the microwave-assisted hydrolysis of mistletoe extract and successfully obtained the flavonoid aglycones-rich extract of mistletoe from glycoside-rich extract (Figure 1B).
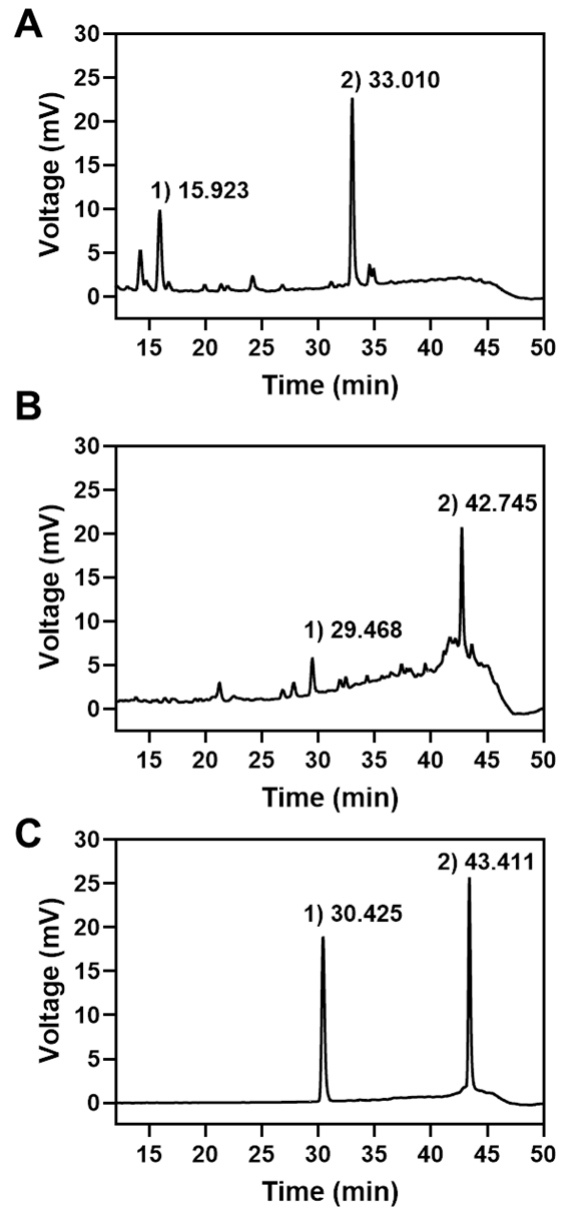

Figure 1. HPLC chromatograms of glycosides-rich (A) and aglycones-rich (B) extract of the mistletoe and a mixture of homoeriodictyol and velutin $(\mathbf{C})$.

Table 1. Parameters used for HPLC analysis.

\begin{tabular}{cc}
\hline Column & TC-C18 $(\mathbf{2 5 0} \times \mathbf{4 . 6} \mathbf{~ m m}$, Particle Size $\mathbf{5} \boldsymbol{\mu m}$, Agilent, USA $)$ \\
\hline Flow Rate & $0.8 \mathrm{~mL} / \mathrm{min}$ \\
$30{ }^{\circ} \mathrm{C}$ \\
Column Temperature & UV $270 \mathrm{~nm}$ \\
Detection & $0.1 \%$ formic acid $(\mathrm{A}), \mathrm{MeOH}(\mathrm{B})$ \\
Solvent & $0-20 \mathrm{~min}, 40 \%-52 \% \mathrm{~B} ; 20-40 \mathrm{~min}, 52 \%-80 \% \mathrm{~B} ; 5 \mathrm{~min}, 40 \% \mathrm{~B}$ \\
Gradient Elution &
\end{tabular}


2.2. Aglycone-Rich Extract from Korean Mistletoe Scavenges ABTS Radical and Inhibits Tyrosinase Activity More Efficiently Than Glycoside-Rich Extract

In order to evaluate the antioxidant activity of the mistletoe extracts, we measured 2,2'-azino-bis-(3-ethylbenthiazoline-6-sulphonic acid) (ABTS) radical scavenging activity within the concentration range from 0.002 to $0.2 \mathrm{mg} / \mathrm{mL}$. Aglycone-rich extract showed concentration-dependent ABTS radical scavenging activity with a maximal scavenging effect of $93 \%$ at $0.05 \mathrm{mg} / \mathrm{mL}$, while glycoside-rich extract showed its maximal scavenging effect of $84 \%$ at $0.2 \mathrm{mg} / \mathrm{mL}$ (Figure 2A).

We also compared the anti-tyrosinase activities of mistletoe glycoside- and aglycone-rich extracts by measuring the amount of dopachrome, which is the product of the tyrosinase-mediated catalytic reaction. Aglycone-rich extract completely inhibited tyrosinase activity at $5 \mathrm{mg} / \mathrm{mL}$, while glycoside-rich extract only inhibited $40 \%$ of tyrosinase activity at the same concentration (Figure 2B). These results suggest that the microwave-assisted deglycosylation of flavonoid glycosides to their aglycones in the mistletoe extract may enhance their radical scavenging activity and inhibitory activity against tyrosinase.

A

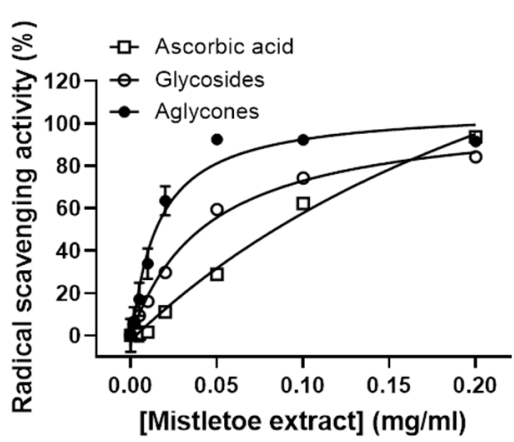

B

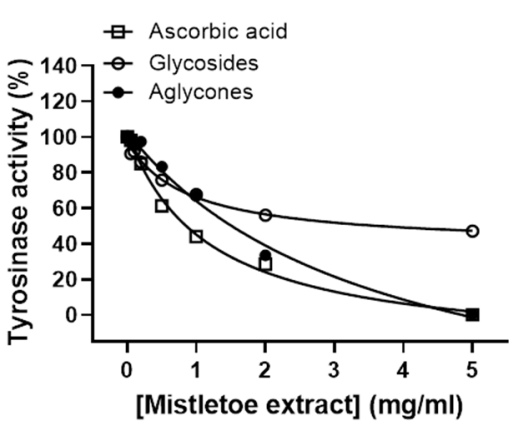

Figure 2. Comparative analysis of glycoside- and aglycone-rich extracts for 2,2'-azino-bis-(3-ethylbenzothiazoline-6-sulphonic acid) (ABTS) radical scavenging and anti-tyrosinase activities. (A) Comparative analysis of ABTS radical scavenging activities of glycoside- and aglycone-rich extracts and ascorbic acid. (B) Inhibitory effects of glycoside- and aglycone-rich extracts and ascorbic acid against tryosinase activity as determined by measuring the amount of dopachrome produced by catalytic reaction of tyrosinase. Results are expressed as the mean \pm SD from three independent experiments.

\subsection{Aglycone-Rich Extract Has a More Potent Inhibitory Effect against Melanogenesis in Zebrafish Embryo Than Glycoside-Rich Extract}

In order to investigate the in vivo recapitulation of our in vitro findings, we first compared the inhibitory effects of the glycoside- and aglycone-rich extracts of mistletoe on melanocyte development in zebrafish embryos at $30 \mathrm{~h}$ post-fertilization (hpf). The melanin synthesis in the early melanocyte development in zebrafish embryos was decreased in a dose-dependent manner by both glycoside-rich extract (Figure 3A) and aglycone-rich extract (Figure 3B). The aglycone-rich mistletoe extract reduced melanogenesis more efficiently than the glycoside-rich extract during melanocyte development, and $330 \mu \mathrm{g} / \mathrm{mL}$ aglycone-rich extract showed a particularly dramatic inhibitory effect (Figure 3 ). This result demonstrated that the microwave-assisted hydrolysis of flavonoid glycosides to their aglycones in the mistletoe extract potentiates the in vivo inhibitory activity on melanogenesis. 
A Glycoside extract (30 hpf)

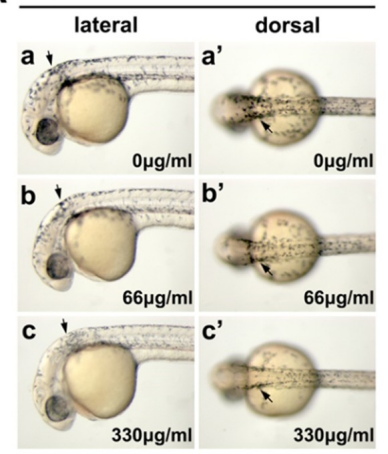

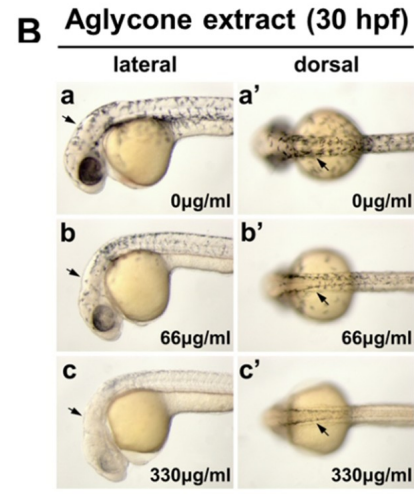

Figure 3. Aglycone-rich extract of mistletoe blocks in vivo melanin synthesis more efficiently than glycoside-rich extract during melanocyte development in zebrafish embryo. Embryos were treated with glycoside extract (A) and aglycone extract (B) at $0 \mu \mathrm{g} / \mathrm{mL}\left(\mathrm{a}, \mathrm{a}^{\prime}\right), 66 \mu \mathrm{g} / \mathrm{mL}\left(\mathrm{b}, \mathrm{b}^{\prime}\right)$, and $330 \mu \mathrm{g} / \mathrm{mL}$ $\left(\mathrm{c}, \mathrm{c}^{\prime}\right)$ in 1\% DMSO/E3 media from five hours post-fertilization (hpf), then imaged at $30 \mathrm{hpf}$. Bright-field images of the embryos are shown in dorsal views $(a-c)$ and lateral views $\left(a^{\prime}-c^{\prime}\right)$. Melanocytes were developed along most of the anterior-posterior axis (arrow) and near the presumptive retinal pigmented epithelium region (arrowhead). Scale bar $500 \mu \mathrm{m}$.

\subsection{Aglycone-Rich Extract of Mistletoe More Efficiently Prevents Melanin Synthesis Than Glycoside-Rich Extract in Zebrafish Embryo}

Next, the capability to maintain the inhibitory activity on in vivo melanin synthesis was examined in this study. Zebrafish embryos were first treated with phenylthiourea (PTU) from 12 hpf, quickly rinsed with 1\% dimethyl sulfoxide (DMSO)/E3 medium at $36 \mathrm{hpf}$, and then treated with mistletoe extracts in 1\% DMSO/E3 medium at different concentrations from 36 to $56 \mathrm{hpf}$. The results indicated that the glycoside-rich extract of mistletoe was slightly effective in inhibiting pigmentation in zebrafish embryos (Figure 4A), but the embryos treated with aglycone-rich extract had a more pronounced effect on preventing melanin synthesis in a dose-dependent manner (Figure 4B). The present data indicate that microwave treatment of the crude ethanol extract of mistletoe increases the in vivo inhibitory effect on melanin synthesis.
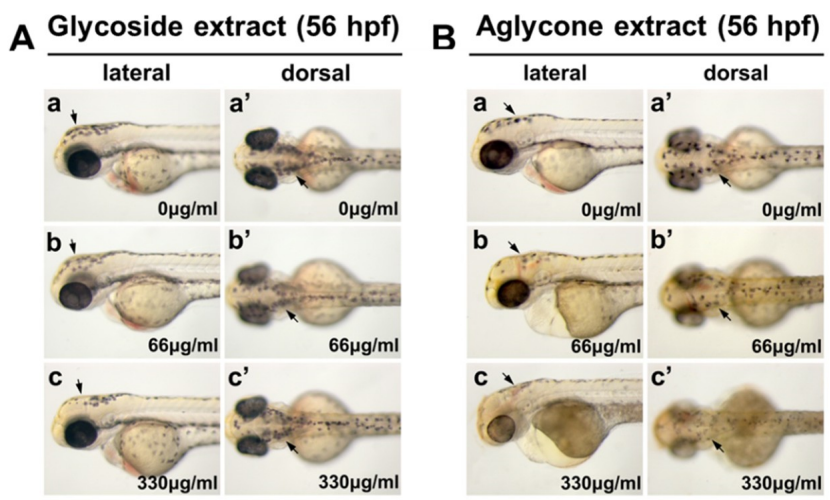

Figure 4. Aglycone extract of mistletoe is a more potent inhibitor on melanin synthesis than glycoside extract in zebrafish embryo. Bright-field images of the embryos are shown in dorsal views $(\mathrm{a}-\mathrm{c})$ and lateral views $\left(\mathrm{a}^{\prime}-\mathrm{c}^{\prime}\right)$. Embryos were pre-treated with $200 \mu \mathrm{M}$ phenylthiourea (PTU) in E3 media from $12 \mathrm{~h}$ post-fertilization (hpf), washed with 1\% DMSO/E3 media at $36 \mathrm{hpf}$, and then exposed to glycoside-rich extract (A) and aglycone-rich extract (B) of mistletoe in 1\% DMSO/E3 media at 0, 66, and $330 \mu \mathrm{g} / \mathrm{mL}$ until $56 \mathrm{hpf}$. Melanocytes were distributed along most of the anterior-posterior axis (arrow) and near the presumptive retinal pigmented epithelium region (arrowhead). Scale bar $500 \mu \mathrm{m}$. 


\subsection{Aglycone-Rich Extract of Mistletoe Is More Biocompatible Than Glycoside-Rich Extract}

Cytotoxicity is a concern for any potential natural compound, and we thus quantitatively measured in vivo the toxicity of the mistletoe extracts in terms of the anti-pigmentation effect using the acridine orange (AO) staining method that visually labels apoptotic cells as green fluorescence dots in live embryos. As an internal control for different sets of the cell death experiments, we used valproic acid (VPA) as a positive control, which consistently causes abundant cell death in zebrafish embryo. The embryos were exposed to the mistletoe extracts from 36 to $52 \mathrm{hpf}$, then subjected to live AO staining and imaging analysis using a confocal microscope. Crude ethanol extract containing flavonoid glycosides led to a dose-dependent increase in cell death (Figure 5A), while there was no significant cell death in the embryos treated with the microwave-irradiated extract containing flavonoid aglycones up to $330 \mu \mathrm{g} / \mathrm{mL}$ (Figure 5B). The data indicate that the pigmentation inhibition of the aglycone-rich extract of mistletoe is not attributable to cell death.
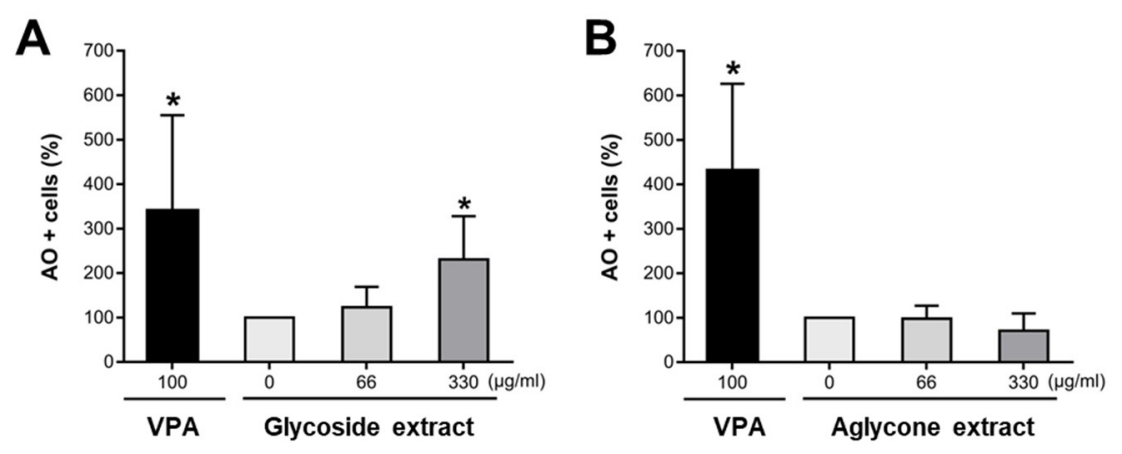

Figure 5. Cytotoxicity tests of glycoside extract and aglycone extract using zebrafish embryo. Embryos were pre-treated with phenylthiourea (PTU) from $12 \mathrm{hpf}$, switched to media containing glycosides(A) or aglycone-rich extract (B) of mistletoe at $36 \mathrm{hpf}$, and then incubated until $52 \mathrm{hpf}$. The live $52 \mathrm{hpf}$ embryos were stained with acridine orange $(\mathrm{AO})$ and imaged using a confocal microscope. Cell death was quantitated by calculating the ratio of the number of acridine orange-positive $(\mathrm{AO}+)$ cells in the embryos treated with each extract over the number of control embryos treated with vehicle. Results are expressed as the mean \pm SD from seven independent experiments, ${ }^{*} p<0.001$.

\subsection{Velutin Plays a Major Role in Anti-Pigmentation Effect of Aglycone Extract}

We identified and isolated velutin (Figure 6), an algycone of homoflavoyadorinin B by ${ }^{1} \mathrm{H}-\mathrm{NMR}$ and ${ }^{13} \mathrm{C}-\mathrm{NMR}$ analyses, from the microwave extract of mistletoe (Figure $1 \mathrm{~B}, \mathrm{C}$ ) and characterized it to investigate its role in the anti-pigmentation effect of the mistletoe extract. Our results showed that velutin efficiently reduced melanogenesis in the melanocyte development of zebrafish embryos (Figure 7A) and maintained an inhibitory effect on melanin synthesis, which was initiated by PTU (Figure 7B) in a dose-dependent manner, mimicking the effect of microwave-assisted flavonoid extract. In addition, according to in vivo cytotoxicity testing through $\mathrm{AO}$ staining of zebrafish embryo, velutin did not cause significant cell death at $300 \mu \mathrm{g} / \mathrm{mL}$ (Figure 7C). This indicates that velutin is generated from the microwave-assisted hydrolysis of homoflavoyadorinin B, a glycoside of mistletoe extract, and may serve as a key aglycone flavonoid acting as a natural compound inhibitor on melanin synthesis. 


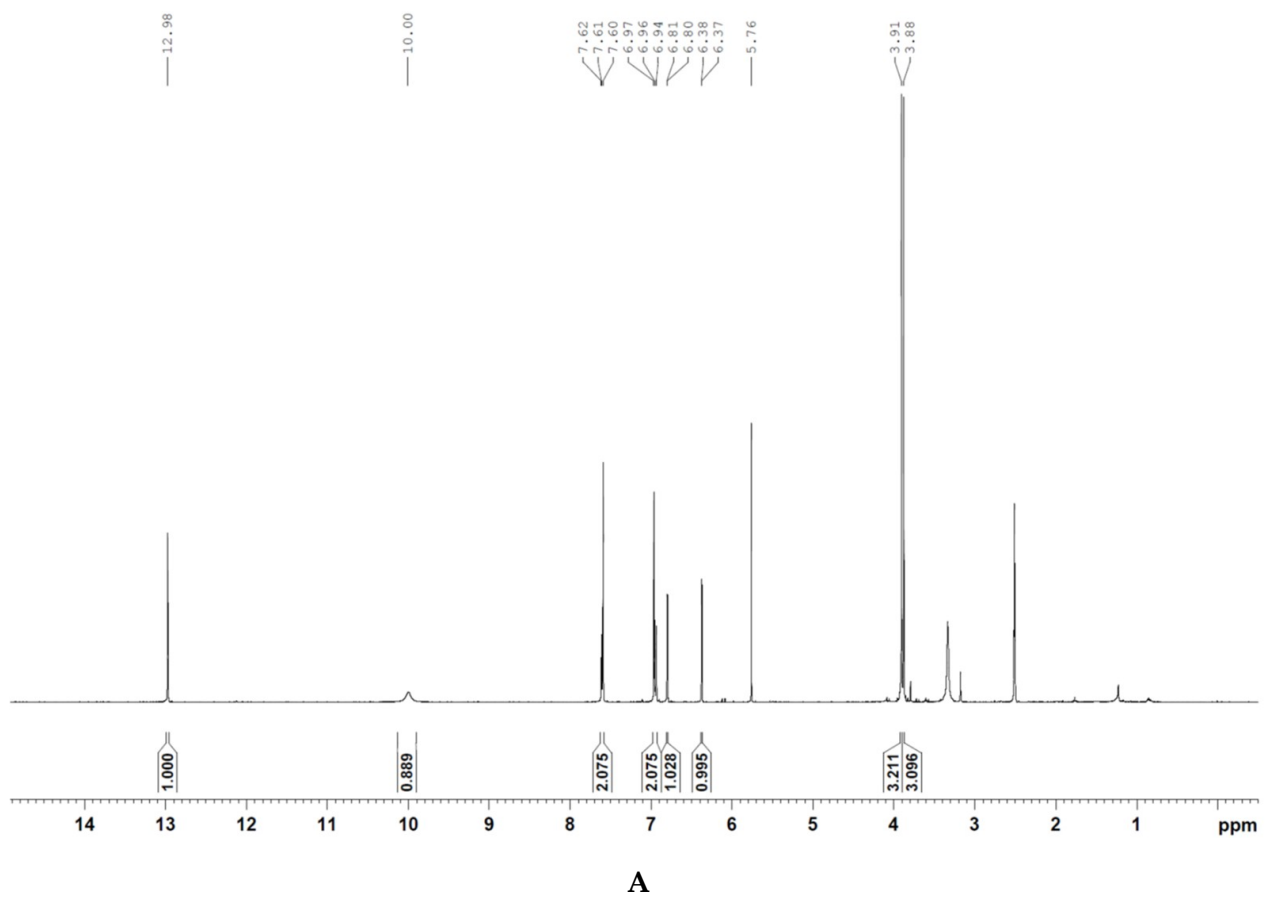<smiles>COc1cc(O)c2c(=O)cc(-c3ccc(O)c(OC)c3)oc2c1</smiles>

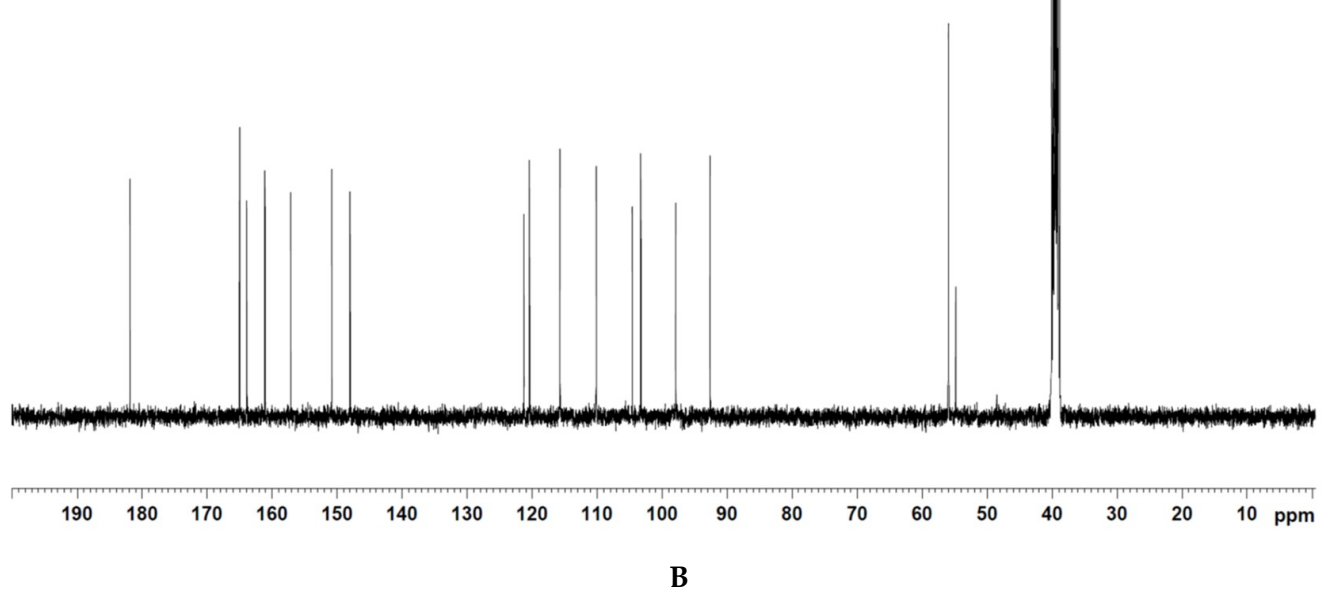

Figure 6. Identification of velutin by ${ }^{1} \mathrm{H}$ NMR (A) and ${ }^{13} \mathrm{C}-\mathrm{NMR}(\mathbf{B})$ analyses. (A) ${ }^{1} \mathrm{H}-\mathrm{NMR}(400 \mathrm{MHz}$, DMSO): $\delta 12.98(\mathrm{~s}, 1 \mathrm{H}), 10.00(\mathrm{~s}, 1 \mathrm{H}), 7.62-7.60(\mathrm{~m}, J=7.24,2 \mathrm{H}), 6.97-6.94(\mathrm{~m}, J=6.44,2 \mathrm{H}), 6.81-6.80(\mathrm{~d}$, $J=2.20,1 \mathrm{H}), 6.38-6.37(\mathrm{~d}, J=2.24,1 \mathrm{H}), 3.91(\mathrm{~s}, 3 \mathrm{H}), 3.88(\mathrm{~s}, 3 \mathrm{H}) .(\mathbf{B}){ }^{13} \mathrm{C}-\mathrm{NMR}(100 \mathrm{M} \mathrm{Hz}, \mathrm{DMSO}): \delta$ $181.9,165.0,163.9,161.1,157.2,150.8,148.0,121.2,120.4,115.7,110.1,104.6,103.3,97.9,92.6,56.01,55.9$. 
A

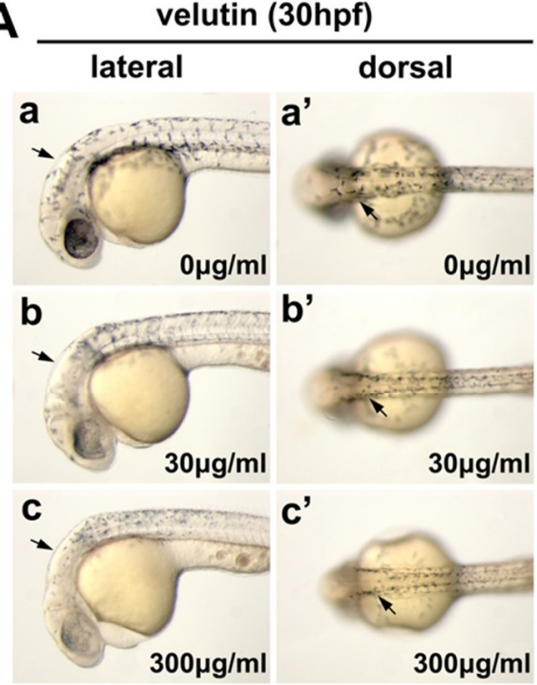

B
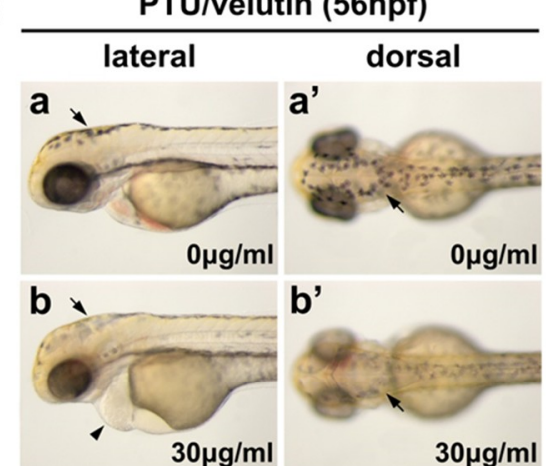

b'

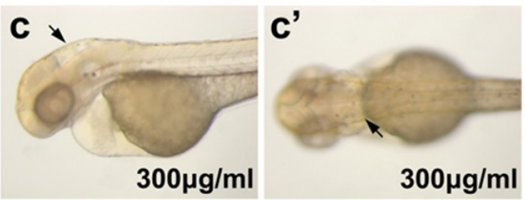

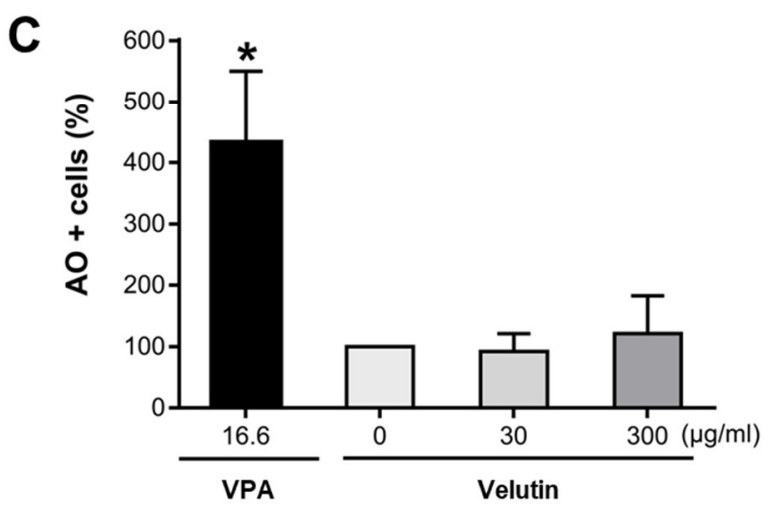

Figure 7. Velutin mimics the anti-pigmentation effect of microwave-assisted aglycone extract of mistletoe in zebrafish embryo. (A,B) Bright-field images of the embryos are shown in dorsal views $(a, b, c)$ and lateral views $\left(a^{\prime}, b^{\prime}, c^{\prime}\right)$. Scale bar $500 \mu \mathrm{m}$. (A) Embryos were exposed to 0, 30, and $300 \mu \mathrm{g} / \mathrm{mL}$ of velutin in 1\% DMSO/E3 medium from five hours post-fertilization (hpf), then the embryos were imaged at $30 \mathrm{hpf}$. Velutin reduces pigment cell development in a dose-dependent manner (arrows). (B) Embryos were pre-treated with $200 \mu \mathrm{M}$ phenylthiourea (PTU) in E3 media from $12 \mathrm{hpf}$, washed with $1 \%$ DMSO/E3 media at $36 \mathrm{hpf}$, and then exposed to 0,30 , and $300 \mu \mathrm{g} / \mathrm{mL}$ of velutin until $56 \mathrm{hpf}$. Velutin efficiently inhibits melanin synthesis in a dose-dependent manner (arrows). (C) Cell death was quantitated by counting the number of acridine orange-positive $(\mathrm{AO}+)$ cells and comparing it to the results for the control with vehicle treatment. There was no significant cell death compared to control embryos. $n=5$ for each treatment, ${ }^{*} p<0.001$.

\section{Experimental Section}

\subsection{Materials}

The mistletoe was collected from an oak tree in Jeongseon in South Korea, then dried under ambient condition before being used in this study, as previously described [16]. 2,2'-azino-bis-(3-ethylbenthiazoline-6-sulphonic acid) (ABTS) and 3,4-dihydroxy-L-phenylalanine (L-DOPA) were purchased from Alfa Aesar (Haverhill, MA, USA). Mushroom tyrosinase, 1-Phenyl-2-thiourea (PTU), and Tricaine methanesulfonate (tricaine) were obtained from Sigma-Aldrich (St. Louis, MO, USA). 


\subsection{Microwave-Assisted Hydrolysis of Flavonoid Glycosides}

Flavonoid aglycones-rich extract was generated through the microwave-assisted hydrolysis of flavone glycosides-rich extract prepared from the mistletoe, in the manner previously described by our lab [23]. Briefly, dried mistletoe (30 g) was pulverized using a pulverizer (HMF-3250s, Hanil electric, Seoul, Korea), then extracted three times with $70 \% \mathrm{EtOH}(300 \mathrm{~mL})$ at $80^{\circ} \mathrm{C}$ for $3 \mathrm{~h}$. The resulting extract was vacuum filtered using Celite 545 (Samchun Pure Chemical Co., Seoul, Korea) and concentrated using a Hei-Vap Advantage rotary evaporator (Heidolph Instruments GmbH \& Co. KG, Schwabach, Germany) to obtain $5.2 \mathrm{~g}$ glycosides-rich $\mathrm{EtOH}$ extract. The microwave-assisted deglycosylation was performed using a microwave reactor, CEM Discover Microwave system (CEM Corporation, Mathews, NC, USA). Briefly, crude EtOH extract (5.2g) was dissolved to a concentration of $200 \mathrm{mg} / \mathrm{mL}$ in acetic acid, poured into a reactor, and irradiated by microwave $(100 \mathrm{~W})$ for $1 \mathrm{~h}$ in a closed system. The temperature of the reactor was kept constant at $120^{\circ} \mathrm{C}$ between each heating. The resulting extract was freeze-dried and kept at $-80{ }^{\circ} \mathrm{C}$ prior to use.

\subsection{HPLC Analyses}

The flavonoid compounds in crude or microwave-irradiated mistletoe extracts were analyzed by HPLC (YL-9100, Young Lin instrument, Anyang, Korea) under the conditions presented in Table 1.

\subsection{Isolation and Identification of Velutin Using Silica Gel Column Chromatography}

The microwave-irradiated crude extract ( $4.4 \mathrm{~g}$ ) was subjected to silica gel column chromatography using gradient dichloromethane-methanol (100:1-50:1) to obtain velutin (37 mg). To confirm that the compound is velutin, ${ }^{1} \mathrm{H}-\mathrm{NMR}(400 \mathrm{MHz})$ and ${ }^{13} \mathrm{C}-\mathrm{NMR}(100 \mathrm{MHz})$ spectra were recorded on an NMR spectrometer (Bruker Advance $400 \mathrm{MHz}$ spectrometer, Bruker Corporation, Karlsruhe, Germany). Chemical shift values $(\delta)$ are reported in parts per million relative to the residual signals of this solvent [ $\delta 2.54$ for ${ }^{1} \mathrm{H}$ (dimethyl sulfoxide-d6), and $\delta 40.5$ for ${ }^{13} \mathrm{C}$ (dimethyl sulfoxide-d6)].

\subsection{Determination of ABTS Free Radical Scavenging Activity}

In order to evaluate the antioxidant activity of flavonoid glycosides and aglycones extracted from Korean mistletoe, the ABTS radical scavenging activity was determined as described in the previous report with some modifications [24]. First, $\mathrm{ABTS}^{+}$radical cation was obtained by reaction between $7 \mathrm{mM}$ ABTS solution and $2.45 \mathrm{mM} \mathrm{K}_{2} \mathrm{~S}_{2} \mathrm{O}_{8}$ solution for $16 \mathrm{~h}$ in the dark. Reaction solution was adjusted with ethanol to yield an absorbance between 0.68 and 0.72 at $734 \mathrm{~nm}$. Then, $100 \mu \mathrm{L}$ of ABTS solution was added to $100 \mu \mathrm{L}$ of each extract in the indicated concentration range from 0.002 to $0.2 \mathrm{mg} / \mathrm{mL}$ and incubated for $5 \mathrm{~min}$ in the dark at room temperature. The absorbance was then measured at $734 \mathrm{~nm}$ using a SpectraMax M5 Multi-Mode microplate reader (Molecular Devices, Sunnyvale, CA, USA). The ABTS radical scavenging activity was calculated as follows:

$$
\text { ABTS radical scavenging activity }(\%)=1-\left(\mathrm{A}_{\text {sample }} / \mathrm{A}_{\text {control }}\right) \times 100 \text {. }
$$

\subsection{Tyrosinase Inhibitory Assay}

The inhibitory effect of microwave-assisted mistletoe extract on tyrosinase activity was assessed based on quenching of fluorescence by dopachrome produced from the catalytic reaction of tyrosinase [25]. Briefly, each $50 \mu \mathrm{L}$ of $0.05-5 \mathrm{mg} / \mathrm{mL}$ flavonoid extract and ascorbic acid was mixed with $50 \mu \mathrm{L}$ of $50 \mathrm{U} / \mathrm{mL}$ mushroom tyrosinase in $50 \mathrm{mM}$ phosphate buffer (pH 6.8) in a 96-well plate and incubated for $30 \mathrm{~min}$ at room temperature. Then, $50 \mu \mathrm{L}$ of $1 \mathrm{mM} \mathrm{L}$-DOPA was added to each well and further incubated for $10 \mathrm{~min}$ at $37^{\circ} \mathrm{C}$. After mixing the solution with $50 \mu \mathrm{L}$ of $400 \mathrm{ng} / \mathrm{mL}$ FITC solution, the fluorescence intensity of the resulting solution was measured using a Synergy H1 Multimode microplate reader from BioTek Instrument, Inc. (Winooski, VT, USA). 


\subsection{Zebrafish Animal Maintenance}

Wild type zebrafish (Danio rerio), $\mathrm{AB}$ strain, was used in this study. The adult zebrafish were maintained in balanced salt water at $27.5^{\circ} \mathrm{C}$ with a $14 / 10$ light/dark cycle. The embryos were raised in E3 medium ( $5 \mathrm{mM} \mathrm{NaCl}, 0.33 \mathrm{mM} \mathrm{MgSO} 4,0.33 \mathrm{mM} \mathrm{CaCl} 2$, and $0.17 \mathrm{mM} \mathrm{KCl})$ at $28.5^{\circ} \mathrm{C}$.

The animal protocols used in this work were evaluated and approved by the Animal Use and Ethic Committee of the Kangwon National University. They are in accordance with Institutional Animal Care and Use Committee (IACUC) guidelines and the National law for Laboratory Animal Experimentation (Law No. 18.611)

\subsection{In Vivo Activity Assay of Flavonoid Extract Using Zebrafish Embryos}

Each flavonoid extract was dissolved in DMSO and diluted in E3 medium for embryonic treatment to final $1 \%$ DMSO. In order to determine the inhibitory activity against melanocyte development, the embryos were treated with glycoside-rich or aglycone-rich flavonoid extract at concentrations of 0,66 , or $330 \mu \mathrm{g} / \mathrm{mL}$ in $1 \%$ DMSO/E3 medium from 5 to $30 \mathrm{hpf}$. Then, bright-field images of the embryos were taken at $30 \mathrm{hpf}$. In each experiment, five embryos were treated with each extract in $2 \mathrm{~mL}$ of E3 medium per well in 24-well plates and incubated at $28.5^{\circ} \mathrm{C}$ until undergoing imaging analysis at $30 \mathrm{hpf}$.

Next, the in vivo inhibitory activity against melanin synthesis was determined as follows: first, 20 embryos were treated with $200 \mu \mathrm{M}$ PTU in $4 \mathrm{~mL}$ of E3 medium in each $30 \mathrm{~mm}$ dish at $12 \mathrm{hpf}$. Next, the embryos were dechorionated at $36 \mathrm{hpf}$ in PTU/E3 medium, washed twice with 1\% DMSO/E3 medium, transferred to 24-well plates, and treated with glycoside or aglycone flavonoid extract in $2 \mathrm{~mL}$ of $1 \% \mathrm{DMSO} / \mathrm{E} 3$ medium from 36 to $56 \mathrm{hpf}$ in zebrafish embryonic development. Then, imaging analysis was performed at $56 \mathrm{hpf}$.

\subsection{Bright Field Imaging of Zebrafish Embryos}

Live zebrafish embryos were anesthetized with $0.02 \%$ tricaine in E3 medium until they were unresponsive to touch, then they were mounted in $3 \%$ methylcellulose for imaging. Bright field images of the embryos were taken using an Olympus SZX16 stereoscope (Olympus, Tokyo, Japan) equipped with an AxioCam GRC camera (Carl Zeiss, Overkochen, Germany).

\subsection{In Vivo Toxicity Test of Flavonoid Extracts Using Zebrafish Embryos}

The toxicity of the flavonoid extracts was evaluated using $\mathrm{AO}$ in the manner described previously [26]. Briefly, embryos were pre-treated with $200 \mu \mathrm{M}$ PTU in E3 medium from $12 \mathrm{hpf}$, washed with $1 \% \mathrm{DMSO} / \mathrm{E} 3$ medium twice at $36 \mathrm{hpf}$. The resulting embryos were further grown in glycosides-rich or aglycones-rich flavonoid extract in 1\% DMSO/E3 medium until $52 \mathrm{hpf}$. Next, the embryos were stained with $3 \mu \mathrm{g} / \mathrm{mL}$ AO in $\mathrm{E} 3$ medium for 20 min with shaking in the dark and washed twice with E3 medium at $25{ }^{\circ} \mathrm{C}$. The embryos were then anesthetized with $0.02 \%$ tricaine/E3 medium and imaged using an LSM700 confocal microscope (Carl Zeiss, Overkochen, Germany). Imaging was performed using $488 \mathrm{~nm}$ Ar-laser excitation and a 415-735 nm emission filter. The number of acridine orange positive $\left(\mathrm{AO}^{+}\right)$cells was counted using the ZEN 2012 program (Carl Zeiss, Oberkochen, Germany). Confocal imaging was conducted at the Korea Basic Science Institute Chuncheon Center.

Cell death was quantitated by calculating the ratio of the number of $\mathrm{AO}^{+}$cells in embryos treated with the flavonoid extracts over the number of control embryos treated with vehicle. The number of $\mathrm{AO}^{+}$cells in the embryos treated with $16.6 \mu \mathrm{g} / \mathrm{mL}$ VPA was used as an internal positive control in each experiment in order to normalize the variation among experiments.

\subsection{Statistical Analysis}

The dopachrome assay results were expressed as the mean \pm SD from three independent experiments. AO staining was used to measure in vivo cell death in zebrafish embryos by calculating the ratio of the number of $\mathrm{AO}^{+}$cells in the embryos treated with the flavonoid extracts over the number 
of control embryos treated with vehicle. The results were expressed as the mean \pm SD from five or seven independent experiments, ${ }^{*} p<0.001$.

Statistical analyses were performed using SPSS 23.0 software (SPSS Inc., Chicago, IL, USA) and Graph-Pad Prism 7.0 (GraphPad Sofware Inc., La Jolla, CA, USA). Five zebrafish embryos per treatment were tested for the effects on melanocyte development and melanin synthesis, and each experiment was repeated for statistical analyses. The difference between the mean values of the control group and the exposed group was analyzed using one-way analysis of variance (ANOVA) followed by a Dunnett's post hoc test. The threshold for statistical significance for all analyses was $p<0.05$ (two-tailed).

\section{Conclusions}

In this study, we obtained aglycone flavonoid extract by the microwave-assisted hydrolysis of glycoside extract from Korean mistletoe under acidic condition, and demonstrated that the aglycone extract scavenges ABTS radicals more efficiently and also has a potent inhibitory effect on melanin biosynthesis without significant in vivo toxicity. Furthermore, we newly isolated and characterized velutin, a single component in the mistletoe extract, and showed that velutin strongly mimics the improved inhibitory activity of microwave-assisted flavonoid extract on melanin biosynthesis. Here, we provided a basis for the successful development of a bio beta natural compound inhibitor from a parasite plant to prevent hyperpigmentation.

Author Contributions: Conceptualization, K.L. and Y.K.; methodology, Y.K.; validation, K.L. and Y.K.; formal analysis, S.-H.J., K.L. and Y.K.; investigation, S.-H.J., J.K., J.E., J.W.C, and H.H.K.; resources, K.L. and Y.K.; writing-original draft preparation, S.-H.J. and J.K.; writing-review and editing, K.L. and Y.K.; visualization, S.-H.J., K.L. and Y.K.; supervision, K.L. and Y.K.; project administration, K.L. and Y.K.; funding acquisition, K.L.

Funding: This work was supported by 2016 Research Grant from Kangwon National University (No. 520160447).

Conflicts of Interest: The authors declare no conflict of interest.

\section{References}

1. Fu, T.; Chai, B.; Shi, Y.; Dang, Y.; Ye, X. Fargesin inhibits melanin synthesis in murine malignant and immortalized melanocytes by regulating PKA/CREB and P38/MAPK signaling pathways. J. Dermatol. Sci. 2019, 94, 213-219. [CrossRef] [PubMed]

2. Wang, L.; Gan, Z.F.; Guo, D.; Xia, H.L.; Patrice, F.T.; Hafez, M.E.; Li, D.W. Electrochemistry-Regulated Recyclable SERS Sensor for Sensitive and Selective Detection of Tyrosinase Activity. Anal. Chem. 2019, 91, 6507-6513. [CrossRef] [PubMed]

3. Rose, P.T. Pigmentary disorders. Med. Clin. North Am. 2009, 93, 1225-1239. [CrossRef] [PubMed]

4. Lee, S.Y.; Baek, N.; Nam, T.G. Natural, semisynthetic and synthetic tyrosinase inhibitors. J. Enzyme Inhib. Med. Chem. 2016, 31, 1-13. [CrossRef] [PubMed]

5. Okombi, S.; Rival, D.; Bonnet, S.; Mariotte, A.M.; Perrier, E.; Boumendjel, A. Discovery of benzylidenebenzofuran-3(2H)-one (aurones) as inhibitors of tyrosinase derived from human melanocytes. J. Med. Chem. 2006, 49, 329-333. [CrossRef] [PubMed]

6. Momtaz, S.; Lall, N.; Basson, A. Inhibitory activities of mushroom tyrosine and DOPA oxidation by plant extracts. S. Afr. J. Bot. 2008, 74, 577-582. [CrossRef]

7. Cazarolli, L.H.; Zanatta, L.; Alberton, E.H.; Figueiredo, M.S.; Folador, P.; Damazio, R.G.; Pizzolatti, M.G.; Silva, F.R. Flavonoids: Prospective drug candidates. Mini Rev. Med. Chem. 2008, 8, 1429-1440. [CrossRef] [PubMed]

8. Cushnie, T.P.; Lamb, A.J. Recent advances in understanding the antibacterial properties of flavonoids. Int. J. Antimicrob. Agents 2011, 38, 99-107. [CrossRef] [PubMed]

9. Xiao, J.B. Dietary flavonoid aglycones and their glycosides: Which show better biological significance? Crit. Rev. Food Sci. Nutr. 2017, 57, 1874-1905. [CrossRef] [PubMed]

10. Jin, X.; Zhang, Z.H.; Sun, E.; Jia, X.B. Beta-cyclodextrin assistant flavonoid glycosides enzymatic hydrolysis. Pharmacogn. Mag. 2013, 9, 11-18. 
11. Zhang, W.L.; Chen, J.P.; Lam, K.Y.; Zhan, J.Y.; Yao, P.; Dong, T.T.; Tsim, K.W. Hydrolysis of Glycosidic Flavonoids during the Preparation of Danggui Buxue Tang: An Outcome of Moderate Boiling of Chinese Herbal Mixture. Evid.-Based Complement. Altern. Med. 2014, 2014, 608721. [CrossRef] [PubMed]

12. Nguyen, V.S.; Cai, S.L.; Feng, T.; Wang, Q.A. Promoting hydrolysis of flavonoid glycosides by microwave irradiation. J. Indian Chem. Soc. 2015, 92, 1579-1582.

13. Nazaruk, J.; Orlikowski, P. Phytochemical profile and therapeutic potential of Viscum album L. Nat. Prod. Res. 2016, 30, 373-385. [CrossRef]

14. Zhang, X.; Yang, L.; Mester, Z. Determination of amino acids in selenium-enriched yeast by gas chromatography mass spectrometry after microwave assisted hydrolysis. Anal. Chim. Acta 2012, 744, 54-59. [CrossRef] [PubMed]

15. Okubamichael, D.Y.; Griffiths, M.E.; Ward, D. Host specificity in parasitic plants-perspectives from mistletoes. AoB Plants 2016, 8. [CrossRef] [PubMed]

16. Park, C.; Kim, J.; Hwang, W.; Lee, B.D.; Lee, K. In vitro anti-tyrosinase activity of viscumneoside III and homoflavoyadorinin B isolated from korean mistletoe (Viscum album). Korean J. Plant Res. 2016, 29, 690-698. [CrossRef]

17. Gerke, T.; SÄTTLER, A. Mistletoe Extracts for Use in Skin Cosmetics. WO2000059464A1, 30 March 1999. Available online: https://patentimages.storage.googleapis.com/39/95/89/454a0948dd3d56/WO2000059464A1 (accessed on 1 March 2019).

18. Chen, W.C.; Tseng, T.S.; Hsiao, N.W.; Lin, Y.L.; Wen, Z.H.; Tsai, C.C.; Lee, Y.C.; Lin, H.H.; Tsai, K.C. Discovery of highly potent tyrosinase inhibitor, T1, with significant anti-melanogenesis ability by zebrafish in vivo assay and computational molecular modeling. Sci. Rep. 2015, 5, 7995. [CrossRef] [PubMed]

19. Lee, Y.R.; Park, J.H.; Castaneda Molina, R.; Nam, Y.H.; Lee, Y.G.; Hong, B.N.; Baek, N.I.; Kang, T.H. Skin depigmenting action of silkworm (Bombyx mori L.) droppings in zebrafish. Arch. Dermatol. Res. 2018, 310, 245-253. [CrossRef]

20. Camp, E.; Lardelli, M. Tyrosinase gene expression in zebrafish embryos. Dev. Genes Evol. 2001, 211, $150-153$. [CrossRef] [PubMed]

21. Strahle, U.; Scholz, S.; Geisler, R.; Greiner, P.; Hollert, H.; Rastegar, S.; Schumacher, A.; Selderslaghs, I.; Weiss, C.; Witters, H.; et al. Zebrafish embryos as an alternative to animal experiments-A commentary on the definition of the onset of protected life stages in animal welfare regulations. Reprod. Toxicol. 2012, 33, 128-132. [CrossRef] [PubMed]

22. Directive 2010/63/EU of the European Parliament and of the Council of 22 September 2010 on the protection of animals used for scientific purposes. Off. J. Eur. Union 2010, 276, 33-79. Available online: https://eur-lex. europa.eu/LexUriServ/LexUriServ.do?uri=OJ:L:2010:276:0033:0079:EN:PDF (accessed on 1 March 2019).

23. Lee, S.; Lee, D.H.; Kim, J.C.; Um, B.H.; Sung, S.H.; Jeong, L.S.; Kim, Y.K.; Kim, S.N. Pectolinarigenin, an aglycone of pectolinarin, has more potent inhibitory activities on melanogenesis than pectolinarin. Biochem. Biophys. Res. Commun. 2017, 493, 765-772. [CrossRef] [PubMed]

24. Hao, K.; Hu, W.; Hou, M.; Cao, D.; Wang, Y.; Guan, Q.; Zhang, X.; Wang, A.; Yu, J.; Guo, B. Optimization of Ultrasonic-Assisted Extraction of Total Phenolics from Citrus aurantium L. Blossoms and Evaluation of Free Radical Scavenging, Anti-HMG-CoA Reductase Activities. Molecules 2019, 24, 2368. [CrossRef] [PubMed]

25. Ma, X.G.; Gao, W.Y.; Halawa, M.I.; Lan, Y.X.; Li, J.P.; Xu, G.B. Lucigenin fluorescent assay of tyrosinase activity and its inhibitor screening. Sens. Actuators B-Chem. 2019, 280, 41-45. [CrossRef]

26. Tucker, B.; Lardelli, M. A rapid apoptosis assay measuring relative acridine orange fluorescence in zebrafish embryos. Zebrafish 2007, 4, 113-116. [CrossRef] [PubMed]

Sample Availability: Samples of the compounds are not available from the authors. 